\title{
Phases of the Diagnostic Journey: A Framework
}

\author{
Linda N Geng ${ }^{1 *}$, Oliver Sum-Ping ${ }^{2}$ and Yong-Jian Geng ${ }^{3 *}$ \\ ${ }^{1}$ Department of Internal Medicine, School of Medicine, Stanford University, USA \\ ${ }^{2}$ Department of Neurology, Division of Sleep Medicine, The Permanente Medical Group, USA \\ ${ }^{3}$ Department of Internal Medicine, McGovern School of Medicine, University of Texas Health Science Center, USA
}

*Corresponding author: Linda N Geng, MD, PhD, Clinical Instructor, Department of Internal Medicine, School of Medicine, Stanford University, Palo Alto, California, USA;

Yong-Jian Geng, MD, PhD, Professor, Department of Internal Medicine, McGovern School of Medicine, University of Texas Health Science Center at Houston, Texas, USA

\begin{abstract}
Diagnostic journeys begin when patients first present to a healthcare provider for their symptoms and end when they receive the correct diagnosis for these symptoms. In many cases, the diagnosis can be made promptly, but patients with rare, complex, or unusual conditions often embark on odysseys spanning years to sometimes even decades in search of a diagnosis. This prolonged process can often result in excessive costs, preventable medical errors and iatrogenesis, as well as feelings of frustration, abandonment and isolation. To address these issues and facilitate discussion, a shared conceptual framework and vocabulary are needed. Here we propose a framework that partitions a patient's diagnostic journey into three distinct phases related to navigation within the healthcare system: The primary encounter, an initial round of referrals, and subsequent evaluations and second opinions. The three phases of the diagnostic journey are interconnected, and each can be further studied and optimized.
\end{abstract}

\section{Keywords}

Diagnosis, Diagnostic journey, Diagnostic odyssey, Undiagnosed, Rare disease, Clinical pathway, Delayed diagnosis

\section{Introduction}

A diagnostic journey begins when a patient first seeks medical care for his or her symptoms and ends when a correct diagnosis is given for these symptoms. An expectation of modern medicine is that if one becomes sick, one can go to the doctor and quickly receive a diagnosis and, hopefully, treatment. While this is indeed the case for many who experience common ailments, there are still many others who have neither smooth nor short journeys. In particular, those with unusual or rare conditions often embark on odysseys spanning years to sometimes even decades in search of a diagnosis. In fact, the average time to diagnosis for a rare condition is reported to be 6 to 7 years $[1,2]$. These patients may be evaluated by numerous different physicians, sometimes eight or more [3], and undergo extensive testing before they reach their final correct diagnosis. During these prolonged diagnostic odysseys, patients and providers often reciprocate frustration. Patients and their caregivers may feel abandoned and isolated [4]. Additionally, there are burdens of cost, intensive utilization of resources, and sometimes preventable medical errors and iatrogenesis along the way [5]. In order to address these issues and improve the process as a whole, a shared conceptual framework and vocabulary are needed. Here we propose a framework that partitions a patient's diagnostic journey into three distinct phases, each of which can be further studied and optimized (Figure 1).

\section{Phase 1: First Encounter with a Healthcare Provider}

Patients enter the first phase when they seek care for their symptoms from a provider, often a generalist such as an outpatient primary care provider or, in more acute conditions, an emergency medicine provider. There are some who never seek care for their symptoms and, therefore, remain in a "Phase 0" (not diagrammed here). Sometimes a patient directly seeks

Citation: Geng LN, Sum-Ping O, Geng YJ (2019) Phases of the Diagnostic Journey: A Framework. Int Arch Intern Med 3:013

Accepted: May 18, 2019: Published: May 20, 2019

Copyright: (C) 2019 Geng LN, et al. This is an open-access article distributed under the terms of the Creative Commons Attribution License, which permits unrestricted use, distribution, and reproduction in any medium, provided the original author and source are credited. 


\section{I A G N O S T I C J O U R N E Y}

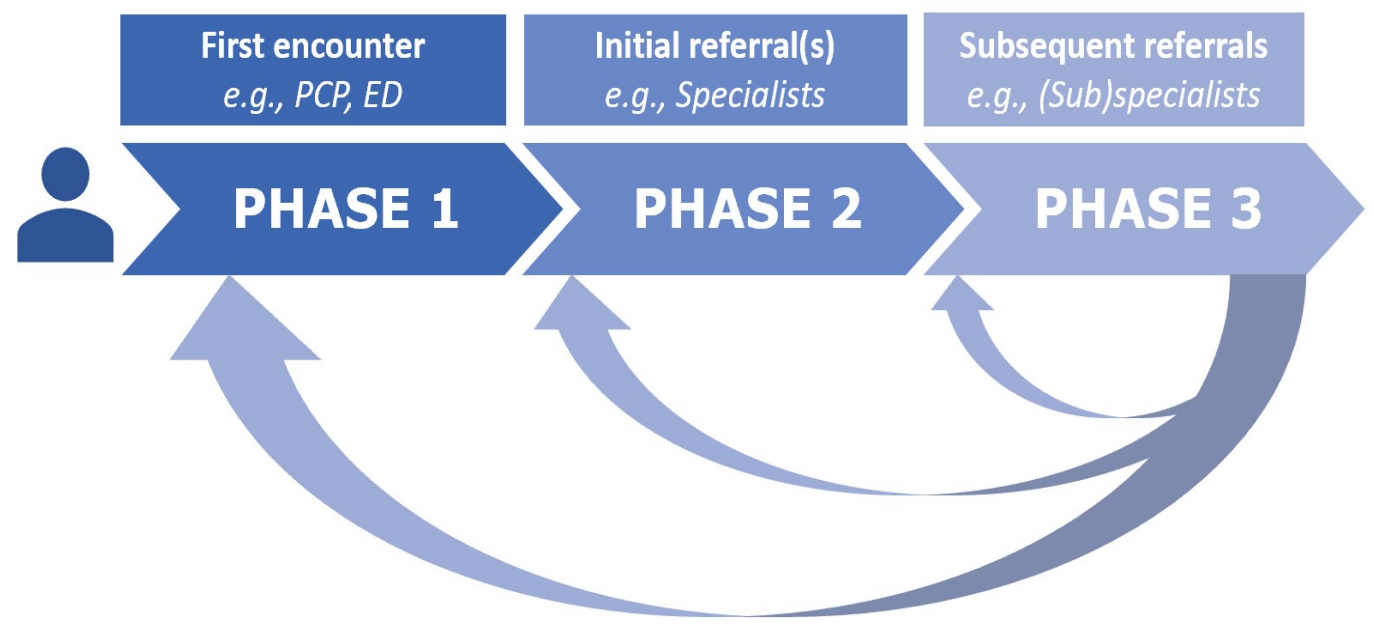

Figure 1: Schematic representation of the three phases of the diagnostic journey: Phase 1) First encounter, when patient is first evaluated for their symptom; Phase 2) Initial round of referral(s), when patient is evaluated by a specialist or specialists either in parallel or in sequence; Phase 3) Subsequent referrals, when patient is evaluated by additional specialists or subspecialists sometimes as second (or third, fourth, etc.) opinions. Progression through the phases is often prompted by persistent or recurrent symptoms in the setting of incorrect diagnosis or lack of a diagnosis. PCP: Primary care provider; ED: Emergency department.

out a specialist to evaluate their condition, which may or may not shorten their journey depending on their choice of an appropriate specialist. For relatively simple and common diagnoses (e.g., pneumonia), the journey may quickly end in one visit with a provider, but for complex or rare diagnoses (e.g., mitochondrial disorder), the journey often continues. Therefore, three outcomes can result from this initial Phase 1 encounter: A) A correct diagnosis is reached and the journey ends; B) No correct diagnosis is reached but patient disengages with the healthcare system for these symptoms, or C) Patient has persistent or recurrent symptoms in setting of either incorrect or lack of diagnosis and, thus, journey continues on to phase 2 .

\section{Phase 2: Initial Round of Referrals}

The second phase of the diagnostic journey typically involves one or multiple evaluations by specialists. For example, a patient presenting with fever of unknown origin may be referred by their primary care physician to a rheumatologist and infectious disease specialist simultaneously or in sequence. This phase may be expedited in inpatient settings where multi-specialty consultation is available, particularly at academic medical centers. However, Phase 2 is more often occurring in the community and at secondary healthcare centers. This phase can be prolonged if there is a lack of local specialists, if the referrals are made in sequence and there is a long wait time, or if specialists disagree with one another about the diagnosis. The outcomes that can result from Phase 2 include: A) A correct diagnosis is reached and the journey ends; B) No correct diagnosis is reached and patient returns to an earlier phase and sent back to the referring provider or, C) No correct diagnosis is reached but patient disengages with the healthcare system for these symptoms, or D) Patient has persistent or recurrent symptoms in setting of either incorrect or lack of diagnosis and, thus, journey continues on to Phase 3.

\section{Phase 3: Subsequent Referrals and Second Opin- ions}

Patients with the most difficult diagnoses tend to enter Phase 3. This third phase of the diagnostic journey typically involves evaluation by additional specialists and subspecialists, often by now occurring the setting of tertiary and quaternary medical centers. These subsequent round(s) of referrals may be made by the patient's primary provider or specialists or the patient themselves. The outcomes that can result from Phase 3 include: A) A correct diagnosis is reached and journey ends, or B) No correct diagnosis is reached and patient returns to an earlier phase, C) No correct diagnosis is reached and patient disengages with the healthcare system for these symptoms. Returning to an earlier phase does not mean the diagnostic complexity has reduced; rather the condition remains an enigma but the challenge of solving the mystery becomes the initial referring provider's again. For unsolved mysteries, innovative programs such as NIH's Undiagnosed Disease Network (UDN) that traverse into the scientific research realm provide opportunities to discover entirely novel diseases [6]. The path by now is hardly linear, but rather circuitous and haphazard. This is the phase where years can pass by, where patients are most likely to "bounced around" from one specialist to another and perhaps fall through the cracks of our fragmented and increasingly subspecialized healthcare system [7]. Patients at the end of this phase may have sought out opinions from 
dozens of physicians at various institutions, across the nation or even internationally.

In conclusion, the diagnostic journey can be conceptualized in three clinical phases, comprising the primary encounter, an initial round of referrals, and subsequent evaluations and second opinions. Most common ailments are diagnosed and addressed within the first two phases, but patients with atypical presentations, complex symptoms, or rare conditions can suffer long diagnostic odysseys and remain in Phase 3 for years or disengage with the healthcare system entirely. How can we shorten the diagnostic journey and, in turn, reduce the burden to patients, providers, and the system as a whole? How do we meet this need within the confines of a given healthcare system? What can we offer patients who have reached the end of Phase 3 but still have no diagnosis? Sustainable solutions to these questions are not crystal clear yet, but programs such as NIH's UDN have demonstrated success in harnessing coordinated, multi-disciplinary and team-based approaches and may serve as prototypes for continued expansion in this arena $[6,8-10]$. Demand for these types of programs has grown and highlights the ubiquity of the problem of patients becoming lost in the diagnostic journey and the gaps of care that need to be filled [11]. Further study and critical scrutiny of the process, raising awareness, and increasing resources to improving the diagnostic journey are greatly needed, and sharing a common conceptual framework of the journey helps to move that important conversation forward.

\section{Disclosures}

None.

\section{Acknowledgements}

The authors thank Dr. Justin Lotfi, Dr. Bryant Lin, Dr. Abraham Verghese, Dr. Megan Mahoney, Dr. Steven
Asch, Sonoo Thadaney, and the Undiagnosed Disease Network team at Stanford, particularly Dr. Euan Ashley and Dr. Matthew Wheeler, for their valuable insights and feedback regarding the conceptualization of this framework.

\section{References}

1. Basel D, McCarrier J (2017) Ending a diagnostic odyssey: Family education, counseling, and response to eventual diagnosis. Pediatr Clin North Am 64: 265-272.

2. Rare disease impact report: Insights from patients and the medical community (2013).

3. Grier J, Hirano M, Karaa A, Shepard E, Thompson JLP (2018) Diagnostic odyssey of patients with mitochondrial disease: Results of a survey. Neurol Genet 4: 230.

4. Baumbusch J, Mayer S, Sloan-Yip I (2018) Alone in a crowd? Parents of children with rare disease's experiences of navigating the healthcare system. J Genet Couns.

5. Hanscom R, Small M, Lambrecht A (2018) Diagnostic accuracy: Room for improvement.

6. Splinter K, Adams DR, Bacino CA, Bellen HJ, Bernstein JA, et al. (2018) Effect of genetic diagnosis on patients with previously undiagnosed disease. N Engl J Med 379: 21312139.

7. Hausmann JS, Lomax KG, Shapiro A, Durrant K (2019) The patient journey to diagnosis and treatment of autoinflammatory diseases. Orphanet J Rare Dis 13: 156.

8. Gahl WA, Wise AL, Ashley EA (2015) The undiagnosed diseases network of the national institutes of health: A national extension. JAMA 314: 1797-1798.

9. Adachi T, Kawamura K, Furusawa Y, Nishizaki Y, Imanishi $\mathrm{N}$, et al. (2017) Japan's initiative on rare and undiagnosed diseases (IRUD): Towards an end to the diagnostic odyssey. Eur J Hum Genet 25: 1025-1028.

10. Armstrong K, Ranganathan R, Fishman M (2018) Toward a culture of scientific inquiry-The role of medical teaching services. N Engl J Med 378: 1-3.

11. Gahl WA, Wise AL, Ashley EA (2016) The undiagnosed diseases program-Reply. JAMA 315: 1904. 